\title{
Civilisations
}

Revue internationale d'anthropologie et de sciences

humaines

$41 \mid 1993$

Mélanges Pierre Salmon II

\section{Les mémorials prestigieux au Royaume Kongo}

\section{Shaje Tshiluila}

\section{OpenEdition}

Journals

Édition électronique

URL : http://journals.openedition.org/civilisations/1704

DOI : 10.4000/civilisations. 1704

ISSN : 2032-0442

\section{Éditeur}

Institut de sociologie de l'Université Libre de Bruxelles

\section{Édition imprimée}

Date de publication : 1 septembre 1993

Pagination : 189-208

ISBN : 2-87263-094-5

ISSN : 0009-8140

\section{Référence électronique}

Shaje Tshiluila, "Les mémorials prestigieux au Royaume Kongo », Civilisations [En ligne], 41 | 1993, mis en ligne le 29 juillet 2009, consulté le 19 avril 2019. URL : http://journals.openedition.org/ civilisations/1704; DOI : 10.4000/civilisations.1704

Ce document a été généré automatiquement le 19 avril 2019

(C) Tous droits réservés 


\title{
Les mémorials prestigieux au Royaume Kongo
}

\author{
Shaje Tshiluila
}

\section{Introduction}

1 Le groupe Kongo fascine autant par son art que par son histoire qui est relativement bien connue.

2 Cette population occupe au Zaïre la région administrative du Bas-Zaïre, mais elle déborde à la fois sur la vaste région du Nord de l'Angola et dans l'enclave de Kabinda ainsi que sur la partie occidentale de la République du Congo.

\section{Historique}

3 Lors de leurs premiers contacts avec la côte occidentale de l'Afrique à la fin du $15^{\text {ème }}$ siècle, les explorateurs découvrent un grand royaume centralisé, le royaume du Kongo, constitué de six provinces annexées et des territoires dépendants (Cuvelier 1946, Vansina 1965). Ultérieurement, ils reconnaîtront l'existence de trois petits royaumes côtiers florissants : le Loango, le Kakongo et le Ngoyo, également habités par des sous-groupes Kongo.

4 Au cours du $14^{\text {ème }}$ siècle, les tendances expansionnistes de Ntinu-Wene ou Nimi-a-Lukeno, fondateur du royaume de Kongo, amènent ses fidèles lieutenants, en l'occurence ses neveux utérins, à conquérir d'autres terres afin d'étendre les frontières du royaume (Montesarchio cité par Cuvelier 1946 : 9-14 ; Vansina 1976 : 32-36).

5 Suivi de quelques parents claniques et de dignes serviteurs, ayant reçu du roi de Kongo le pouvoir de régner et d'établir des chefs subalternes, Ne Mboma, neveu du roi du Kongo et en l'occurence Chef du clan Mboma Ndongo, quitte Mbanza Kongo et se dirige vers l'Ouest. La tradition rapporte qu'après un arrêt à Vunda dia Kongo, il traversa le fleuve Zaïre à Nsanda Nzondo et occupe sur la rive gauche du fleuve la chefferie de Boma qui 
dépendra dorénavant directement du royaume de Kongo. Cuvelier précise que cette chefferie s'étend jusqu'à la Lukunga et comprend surtout l'île de Boma aussi appelée île des Princes (Cuvelier 1953a, 8 ; Maes 1938 : 129).

6 Dans la même vague de déplacements, Ne Mbinda, également investi Chef par le roi Ntinu Wene, passe le fleuve Zaïre à Tadi dia Mpambu en amont de Matadi. Il étend son autorité sur le territoire compris entre la Mao et la Lukunga. Habitées par les Bamimbala, ces terres font partie du royaume de Kongo, du moins les chefferies de Mbinda et de Nsanda (Cuvelier 1953a: 8).

7 Deux autres chefs, Manzadi et Mambungu, se dirigent vers le royame du roi de Kakongo. Passant par les îles de Mateba, ils débarquent sur le territoire royal. A l'époque, ce royaume est également tributaire de celui de Kongo (Cuvelier 1953a : 7; Vansina 1976: 32).

8 Avec l'autorisation du roi Makongo de Kakongo, Manzadi et Mambungu bénéficient d'une cession de terres. Car, suivant les coutumes et usages, la terre appartient aux premiers occupants (Cuvelier 1953b: 79; Ekholm 1972: 71-82). Le droit à l'installation et à l'exploitation s'obtient moyennant une somme (biens matériels, esclaves...) équivalent à la valeur présumée du terrain minutieusement délimité (Delcommune 1922 : 44).

9 Unanimement reconnu comme acquéreur, le nouveau propriétaire, s'il dispose des moyens matériels suffisants, cherchera à être investi du pouvoir cheffal sur ses nouvelles terres. Par la suite, il devra cependant faire preuve de son allégeance en payant régulièrement son tribut et en assistant, selon les besoins, le grand chef propriétaire des terres (Vansina 1976 : 36).

10 Manzadi et Mambungu s'installent donc sur les terres du roi de Kakongo ; le premier sur le littoral entre la Lukunga et la Lusona Mwana Mbola, le second remonte vers le Nord et occupe le territoire compris entre ces mêmes rivières. "Toute la partie du territoire comprise approximativement entre la rivière Lusona-Mbola et la frontière portugaise de l'enclave de Cabinda à l'Ouest, la voie de chemin de fer du Mayumbe à l'Est, le fleuve Zaïre au Sud, la rivière Lukula au Nord, relevait autrefois directement ou indirectement du royaume de Kakongo" (Archives administratives de Boma ; Boone 1973 : 72).

11 Les aléas de l'historie font que les différents groupes Bakongo ba Boma se retrouvent partagés entre deux royaumes séparés par la rivière Kalamu. A l'Est, le royaume de Kongo avec ses tributaires et à l'Ouest, le royaume de Kakongo avec ses chefferies vassales (Fotems 1960: 80), chacun ayant une manière particulière de magnifier ses chefs prestigieux par des monuments remarquables.

12 En 1932, l'administration territoriale coloniale essaye de regrouper les petites subdivisions Bakongo ba Boma qui possèdent un profond lien historique. De la concertation entre l'administration coloniale d'une part et le pouvoir traditionnel d'autre part, naquit l'idée de créer les chefferies de Manzadi et de Bungu englobant respectivement les anciens groupements suivants; pour Manzadi : Shika Shenga, Kanzi, Zambi, Sanzi, Katala et Loango Batshi; pour Bungu: Bungu, Lusanga, Mahulu, Makai Gubunga et Seke dia Bungu (Archives Administratives de Boma).

Les enquêtes entreprises par la suite dans la région ont abouti à adjoindre aux groupements déjà créés d'autres petits ensembles dont les chefs recevaient autrefois l'investiture soit directement du roi de Kakongo soit indirectement de ses vassaux Mambungu ou Manzadi. Ainsi, par l'Arrêté nº 416/2040 AIMO du 15 juillet 1942, est fondée 
la collectivité de Boma Bungu incluse dans la zone de Moanda et dépendante de la sousrégion de Boma.

Cette collectivité de Boma-Bungu se trouve actuellement à $10 \mathrm{Km}$ du centre urbain de Boma. Les données que nous avons récoltées situent au même endroit le petit royaume de Bungu maintes fois mentionné dans les sources historiques écrites (Cuvelier 1946; Vansina 1965,1976 ) et considéré comme le lieu d'origine du groupe de conquérants qui ont fondé le grand royaume de Kongo.

Quant à la dénomination de Vungu que nous trouvons chez Cuvelier (1946: carte) et qui est reprise par Ekholm (1972: carte), nous pensons qu'il peut s'agir soit de deux formes du même nom, soit tout simplement de variantes régionales. En effet, le "B" de Bungu et le "V" de Vungu sont des consonnes labiales sonores et l'on peut facilement passer de l'une à l'autre. La seule différence réside dans le mode de formation, B étant une occlusive et $\mathrm{V}$ une fricative. également dénommée Binda.

La composition de la population rurale de cette collectivité est en majorité Bakongo ba Boma. Bien que Fortems (1960: 19-22) affirme "que les secteurs de Bungu et de Boma soient surtout peuplés de Kakongo". Les recenseurs de 1983 sont éloquents à ce sujet. Sur les 20.880 habitants qui forment la population totale, 590 seulement sont étrangers, il s'agit en général de riverains Assolongo venus d'Angola.

Un autre recensement, antérieur à celui-ci, établi pour la même collectivité pour décembre 1981, présente d'une façon détaillée l'ensemble des localités et des villages Bakongo ba Boma, leur date de création ainsi que la répartition de la population par catégories.

\section{Contacts et échanges}

19 La proximité du fleuve Zaïre, navigable sur le tronçon Matadi-Océan Atlantique, et l'accessibilité des rives de l'océan à la navigation ont ouvert assez tôt la région au commerce avec l'extérieur. Les relations suivies établies dès le premier voyage de Diego Cao en 1432 entre le Portugal et le royaume de Kongo ont débouché sur des échanges commerciaux qui demeureront longtemps fructueux.

Dès la fin du $15^{\text {ème }}$ siècle, s'installa entre l'Europe, l'Afrique et l'Asie, un commerce triangulaire qui, au fil des années, drainera des marchandises nouvelles. Les épices des Indes transitent par les ports du royaume de Kongo avant de passer en Europe. Les esclaves, à peu près 35.000 par an (Curtin 1969 : 4-8, Miller 1975 : 135-176) dans la seconde moitié du $17^{\text {ème }}$ siècle, vont grossir la main-d'oeuvre dans les plantations européennes d'Amérique. Le nombre d'esclaves exportés à partir des côtes occidentales de l'Afrique atteindra le chiffre exorbitant de 80.000 par an (Curtin et Vansina 1964:185-208; Curtin 1969) dans la seconde moitié du $18^{\text {ème }}$ siècle.

21 Mais, à partir de 1830, la demande d'esclaves va décliner très rapidement pour céder la place à la demande d'ivoire et de cire (Miller $1970: 174-201)$. Le caoutchouc, les arachides, les sésames, l'huile de palme, l'ivoire et le café achetés à Boma sont vendus sur les marchés européens (Delcommune $1922: 45$ ). 

d'abord de Magapatam en Inde au $16^{\text {ème }}$ siècle, puis des ateliers verriers de Venise et d'Allemagne à partir du $17^{\text {ème }}$ siècle, sont utilisées sur cette côte occidentale de l'Afrique pour acheter d'abord les esclaves, puis l'ivoire, l'or, le cuivre... (Van der Sleen 1958: 211-212 ; Monteiro 1968 : 191). facilement certains articles de luxe en provenance d'Europe: chapeaux, boissons alcoolisées, faïences anglaises, hollandaises ou allemandes, etc... Il n'était pas rare qu'un chef Kongo reçoive un cadeau somptueux. C'est ainsi qu'en 1643, le comte de Nassau a offert au comte de Sohio, Daniel da Silva, un fauteuil, un manteau et un glaive (Jadin 1975: 661). Deux ans plus tard, ces objets remarquables attirent l'attention des missionnaires envoyés par le Saint-Siège qui relatent ainsi leur première entrevue avec le comte de Sohio : "Assis sur un fauteuil de damas et brocard fin. Près de lui, était une très grande multitude de nobles et fidalgos qui l'entouraient et restaient agenouillés. Du côté droit, un page portait une épée d'apparat bien ornée et à gauche, un autre portait le sceptre (Jadin 1975 : 661).

L'usage des objets d'apparat ainsi que l'échange de cadeaux remontent aux premiers contacts. Un passage de la Relatione de Pigafetta et Lopez en donne une bonne illustration par le récit détaillé consacré à l'habillement de $\mathrm{D}$. Catarina, épouse de $\mathrm{D}$. Alvaro $1^{\mathrm{er}}$, roi de Kongo de 1468-1487 : "Sa tête était complètement rasée (comme au rasoir) et couverte d'un bonnet de velours cramoisi de Milan; elle portait un vêtement d'étoffe noire, avec des revers de velours rouge, et des manches avec leurs revers à la manière des vêtements que portent les auditeurs..." (Cuvelier et Jadin 1954 : 138). Et, lorsqu'ils font la description du royaume de Kongo et des contrées environnantes, Pigafetta et Lopez mentionnent également l'arrivée, au début de l'année 1491, des navires qui avaient à leur bord les premiers évangélisateurs du Kongo ainsi que les présents qu'envoyait le roi du Portugal Jean II au roi de Kongo, "les vêtements sacerdotaux, les ornements de l'autel, les crucifix, les tableaux représentant des saints, les bannières, le gonfalon et le reste" (Pigafetta et Lopez, traduit et annoté par Bal $1963: 84$ ).

Ces rapports suivis se caractérisent assez tôt par l'envoi d'émissaires et même par l'échange d'ambassades (Ibidem : 80-197).

Les traces de ces contacts transparaitront dans les monuments funéraires.

\section{Localisation}

\section{Population}

Nous nous étions d'abord intéressé aux Bakongo ba Boma qui vivent aux alentours de la ville de Boma, dans la sous-région de Boma et qui produisent les deux types de monuments funéraires, à savoir les sculptures en pierre et les céramiques.

Les enquêtes de terrain et le dépouillement des documents écrits (Verly 1955; Cornet 1981) ont élargi le champ d'investigation en y incluant les Mboma et les Kakongo du Zaïre ainsi que les Assolongo et Mboma du Nord de l'Angola.

O. Boone (1973 : 40-169), qui subdivise les Kongo en trois grands groupes, les occidentaux, les centraux et les orientaux, auxquels elle rattache les différents sous-groupes kongo, mbata, ndibu, woyo..., ne mentionne nulle part les Bakongo ba Boma. Mais, dans le 
paragraphe concernant la démographie, l'auteur cite une étude de 1970 consacrée à l'évolution numérique des populations du district du Bas-Congo où les Bakongo ba Boma sont confondus ave les Mboma, leurs voisins orientaux (Boone 1973 : 115). Elle en conclut que le Mboma sont très probablement des Kongo originaires de la région de Boma, et elle ajoute qu'"aucune étude publiée ne le situe avec quelque précision" (Boone 1973 : 116).

\section{Monuments funéraires}

Les sculptures en pierre et les céramiques richement décorées étaient utilisées comme monuments commémoratifs érigés au départ à l'intention des personnalités investies de pouvoir et par la suite également à la mémoire des hommes suffisamment riches.

signalé l'existence d'une sculpture en pierre avec une telle diversité de thèmes et d'une grande céramique énigmatique que dans la sous-région de Boma au Zaïre et dans le Nord de l'Angola.

\footnotetext{
monuments à la nature du sol. La région montagneuse de formation ancienne, située entre Boma et Matadi, qui se prolonge au Nord de l'Angola jusqu'à la hauteur de Nzeto (Ambizete), a favorisé l'éclosion et l'essor de la sculpture en pierre. Le sous-sol de cette région est constitué de roches dures (Cahen et Lepersonne 1948: 66) appartenant au complexe critallophyllien.

L'étude menée dans la région met en évidence le lien étroit qui unit les différents types de
} 
autre part, la zone littorale comprise entre la côte et les premiers reliefs de la chaîne cristalline est riche en argile propice au façonnage des céramiques. Délimitée par la Lukunga à l'Est, la Lukula au Nord, la Lusona-Mwana Mbola au Sud-Ouest et le fleuve Zaïre au Sud, cette région comporte des plages d'étendues très variables dont les sols sont formés par des limons sableux et argileux qui se sont déposés jadis sur les rives du fleuve et à l'embouchure de ses tributaires (Meulenberg 1949 : 32).

\section{Signification}

Des chercheurs qui s'étaient antérieurement intéressés à ce sujet avaient considéré forme et fonction de ces monuments sur des paliers différents. Les uns s'étaient contentés de la dimension historico-thématique, d'autres s'étaient arrêtés à une classification stylistique sans aucune considération des variables pertinentes.

Notre démarche qui associe les traditions régionales à une analyse morphologique rigoureuse et qui s'est appuyé selon les cas sur des documents écrits, historiques ou linguistiques nous a amené à repenser certaines interprétations classiques et à déduire que la forme de ces objets fascinants est liée à leur signification dans la mesure où elle nous introduit dans l'univers symbolique kongo.

En effet, lorsque la pierre provient du massif montagneux (mont de cristal), la céramique, également utilisée dans un contexte funéraire, provient elle de la zone littorale. Elle rejoint cependant la sculpture en pierre par ses proportions et par leur origine, les ravins et l'eau, considérés comme habitat par excellence des esprits de la nature - bisimbi.

Lorsque la grande céramique est ornée de figurines, celles-ci sont empreintes du même réalisme que les sculptures en pierre. Sa forme, proche de celle de la défense d'éléphant, suggère que la céramique a servi de substitut à cette dernière.

Ainsi, dans cette société matrilinéaire d'agriculteurs sédentaires où le rôle de l'oncle maternel, Chef du clan et officiant du culte familial, est continuellement mis en évidence, la consécration d'un pouvoir politico-religieux est liée au rattachement à un droit foncier transmis en héritage par la voie des femmes.

Ces mémorials finissent par apparaitre ici comme des sinsu, diront nos informateurs, c'est-à-dire des signes, des symboles qui poursuivent un triple but :

- marquer la terre à la manière des safoutiers (Dacryodes buettneri) et des palmiers élaïs

(Elaeïs ginneensi) qui délimitent un domaine foncier ;

- embellir et identifier la tombe d'un aîné ;

- et enfin transmettre aux générations futures le souvenir d'un prestige qui rejaillit sur toute la descendance.

Les monuments modernes en ciment perpétuent cette tradition.

Cette conception est à rapprocher de la mentalité de l'époque où nous situons le rayonnement des monuments funéraires.

47 En effet, dans la seconde moitié du $17^{\text {ème }}$ siècle, écrit Hilton (1985: 103), l'action des missionnaires capucins fait apparaître une prolifération d'églises aussi bien dans la capitale du royaume de Kongo à Mbaza Kongo que dans les provinces à Mbanza Soyo et à Mbanza Nsundi. Ces églises sont appelées nzo à nkisi, maison de nkisi ou de tombes. A Mbanza Kongo, en effet, les églises servaient surtout de lieu d'inhumation pour les rois et on y rendait un culte aux morts grâce auquel le roi en place légitimait son pouvoir en 
rappelant ses liens avec ses prédécesseurs défunts. Dans les provinces, par contre, les églises ne servaient pas de lieux de sépulture car les gouverneurs dépendaient du pouvoir central qui les mettait en place ou les démettait selon son bon vouloir. Mais, lors du passage des capucins, les gouverneurs utilisaient les églises pour exalter leur position de contrôleurs du pouvoir.

Précisons encore que les gouverneurs de province étaient, suivant les usages et coutumes, enterrés dans leur localité d'origine. Ainsi, les églises jouaient-elles à Mbanza Kongo le rôle qui revenait aux cimetières des grands chefs dans les provinces.

Qu'on se trouve donc à Mbanza Kongo ou dans les anciennes provinces de Soyo et de Mbamba, les monuments funéraires sont considérés comme des signes bisinsu qui identifient en glorifiant. Le mot "glorifier" ainsi que ses synonymes "initier", "consacrer" et "couronner" (Bentley 1895: 434-5; Butaye 1909: 266; Laman 1936: 993; Swartenbroeckx 1973 : 659) renvoient au terme Bitumba qui désigne les statues en pierre.

Cependant on peut nous objecter que les mots "tumba" et "sinsu" sur lesquels nous nous appuyons pour déceler la signification profonde de ces mémorials sont des mots d'emprunts aux langues romanes.

51 Mais, une reconstruction linguistique de ces mots dont les radicaux verbaux se retrouvent dans presque toutes les zones de langue bantoue, permet d'attester leur origine ancienne et même proto-bantoue.

52 La langue bantoue kongo est classée par Guthrie (1948: vol I à IV) dans la zone H 16. On peut relever l'exemple du mot "tumba". Les dictionnaires consacrés aux différents dialectes kongo donnent pour le verbe transitif kutumba aussi bien le sens de "to make a great" make much, que celui de: "to initiate (into fetisch mysteries), to conservate, to install in an appoint to an office, to invest with (Bentley $1895: 434-5$ ).

Dans le dictionnaire de Laman (1936 : 993-4), le radical - tumb - signifie : "introduire dans, initier quelqu'un, promouvoir, couronner...", tandis que le sens du thème - tumba - est : "ressemblance, image, photographie, statue, figure de porcelaine (pour les tombes)".

Enfin, Swartenbroeckx (1973: 659) donne pour le verbe kutumba le sens de "consacrer, initier, introniser un chef, ordonner prêtre.

55 Lorsque nous considérons la langue buma en zone B74, voisine nord de la zone $\mathrm{H}$, nous constatons que le verbe transitif kututna qui apparaît sans la consonne bilabiale - b - a le sens de "soulever, lever".

56 Cependant, en zone holoholo D28, Coupez (1955: 151) mentionne deux radicaux à extension - tumbuk - et - tumbul - dont le premier intransitif a le sens de "voler" (en parlant d'un oiseau) et le second transitif signifie "soulever".

57 A l'Est, en zone J13, le verbe kutumba est mentionné en ganda avec comme signification "se lever, s'élever". Il s'enrichit aussi du sens de monter, gonfler" (Kitchning and Blackledge 1953 : 174).

58 Ce verbe se rencontre également en Rundi (J62) sous la forme gutumba et se traduit par "être gonflé", "être enflé" tandis que les thèmes simutumba et agatumba signifient "colline, mont" pour le premier, "monticule, tertre et mamelon" pour le second (Rodegem 1970 : 510).

59 Le champ sémantique du radical - tumb - apparaît ainsi bien élargi et couvre à la fois le sens "d'initier, consacrer, conférer, couronner, élever" et celui de "soulever, lever, rendre important, vanter ses mérites, louanger, glorifier". 
60 On connaît également des dérivés tels que "avoir des excroissances, gonfler, crever" en parlant des "boutons, abcès, cadavre" ou "bourgeonner, saillir" ou encore "s'envoler" en parlant d'un oiseau ou "rebondir" lorsqu'il s'agit d'une balle.

61 En kiguyu, zone E51, le verbe kutumba conserve le sens de "isolate, set apart" (Benson 1964 : 478).

Lorsque nous nous tournons vers le Sud-Ouest, nous trouvons en kwamyama, zone R21, le verbe kutumba avec le sens de "surélever, exhausser, majorer" (Brincker 1891 : 73). Et, en herero, R31, ce même sens se maintient, le verbe kutumba se traduisant par "surélever, exhausser (Irle 1917 : 112).

Les zones centrales $\mathrm{K}$ et $\mathrm{L}$ livrent également des données abondantes.

En zone pende, K52, le verbe transitif kutumbuisa apparaît avec le sens de : "faire parler de quelqu'un", "faire citer le nom de quelqu'un". Il peut être rapproché du thème tumba "talus, monticule" (Gusimama 1972 : 207).

65 De plus, dans la même zone en luvale, K14, le verbe kutumbumuka conserve le sens de : "become distinguished or designated from others, as by superior accomplishments" tandis qe le thème - tumba - signifie : "magic, medecine, charms" (Hotron 1953 : 356-7).

Dans la zone Luba, L31, le verbe intransitif kutumba signifie "être connu, renommé, célèbre, honoré" tandis que le verbe kutumbisha à extension causative se traduit par "faire connaître, célébrer, magnifier, glorifier, rendre hommage à". Le verbe kutumbika a pour sa part le sens de mettre en tas, tandis que la forme intransitive kutùmbuka signifie : "passer au-dessus, dominer, s'élancer, être lancé, être haut, élancé" (De Clerc 1960 : 374-5).

67 Dans la même zone, en sanga, L35, le verbe kutumba apparaît avec les nuances relevées précédemment. Il est traduit par "être haut, élevé" ou par "sortir, pousser" ou encore par "devenir important, puissant, supérieur, grandir en influence ou en valeur (Missions bénédictines $1950: 173-4)$.

68 Egalement dans la partie centrale, en ila, zone M63, kutumba signifie: "rassembler, recueillir" tandis que kutumbika à extension - ik - impositif a le sens de "donner à une personne tout son pouvoir" (Smith 1907 : 472).

69 En Tumbuka-Tonga, N21, le verbe kutumbika se traduit par "estimer, honorer, respecter" et le thème - tumbika - renvoie à : "honneur, respect" (Turner 1952 : 147).

70 Dans la zone P21 en yao, le verbe kutumbuka est attesté aussi bien avec le sens de "monter en épi" grandir, s'élever" qu'avec celui de "crever, éclater, faire explosion" (Viana $1961:$ : 167).

71 A l'extrême sud, le verbe tumbuka est présent en shona, zone S10, avec le sens de "bourgeonner, éclater, s'ouvrir, s'élever" tandis que le thème tumba signifie: "un personnage riche, important" (Hannan 1974 : 686).

72 La reconstruction du thème tumba à laquelle nous aboutissons, détermine son origine ancienne et même proto-bantoue. Le radical verbal - tum - est en effet attesté dans presque toutes les zones des langues bantoues, aussi bien à l'Est et l'Ouest qu'au Centre et au Sud.

73 Il semble donc que le substantif tumba convient comme dénomination aux deux types de mémorials (sculpture en pierre et céramique), parce qu'il rend bien leur signification première d'objets qui élèvent, qui glorifient. 
74 Le thème ntadi employé pour la statue en pierre est une métonymie qui désigne l'objet achevé par la matière dans laquelle il est fabriqué, tandis que le substantif diboondo qui signifie la céramique funéraire renvoie plutôt à la technique de modelage dibuumbi.

\section{Conclusion}

La tradition nous livre par un langage métaphorique la fonction de ces statues qui glorifient et élèvent (tumba) en les comparant aux défenses d'éléphants et en les considérant comme des signes, des symboles à rapprocher des photographies.

76 En effet, telles des photographies, ces statues-signes sont destinées à embellir et à identifier certaines tombes le plus longtemps possible et cela à la manière prestigieuse des défenses en ivoire, éléments de la nature, considérées métonymiquement comme imposantes puisque provenant d'un animal réputé pour sa ténacité et sa force. Ainsi, pour assurer l'identification d'une tombe prestigieuse, la synthèse nature - culture exprimée par la statue en pierre prend toute son importance.

Ce sont nos informateurs eux-mêmes qui ont établi la comparaison avec des photographies. Dans le même esprit, de Heusch (1963 : 103) parlait jadis de "portrait" à peu près dans le même contexte. Il écrivait "là où le pouvoir central s'affirme, la volonté aristocratique (en partie désacralisée) de perpétuer le souvenir aboutit à un art du "portrait" royal".

78 On peut objecter que le "portrait" est inadéquat dans tous les cas puisque visiblement, le sculpteur se réfère à un archétype. Mais c'est la fonction, non la forme, qu'il faut apprécier ici. Les célèbres bustes d'Ife comme les statues kuba "re-présentent, au sens fort du terme". Et, il arrive que la fonction commémorative et la fonction religieuse se combinent...

79 Ainsi pour glorifier cette personnalité importante qui est un chef consacré, il s'avère déterminant qu'il soit représenté sous son jour le plus avantageux, entouré de sa cour ou d'éléments qui y font allusion.

80 Cette cour tournée vers l'extérieur est destinée au monde des vivants. Elle a son pendant dans une autre cour placée à l'intérieur de la tombe et chargée d'accompagner et de servir le Chef dans le village des ancêtres.

81 Cette dernière cour se présente aussi pleine de grandeur et on y rencontre mêlés, esclaves et épouses, objets usuels et de prestige.

\section{BIBLIOGRAPHIE}

BAL, W. - 1963 - Description du Royaume de Congo et des contrées environnantes par Filippo Pigafetta et Duarte Lopez (1591), Louvain, Ed. E. Nauwelaerts ; Paris, Ed. Béatrice Nauwelaerts.

BENSON, T.G. - 1964 - Kikuy-English dictionary, Oxford, Clarendon Press. 
BENTLEY, W.H. - 1895 - Dictionary and Grammar of the Congo language as spoken at San Salvador, London, Baptist Missionary Society (1 ${ }^{\text {ère }}$ éd. 1887).

BOONE, O. - 1973 - Carte ethnique de la République du Zaire : quart Sud-Ouest, Tervuren, A.M.R.A.C., série in $8^{\circ}$, Sciences Humaines, 78.

BRINCKER, P.H. - 1891 - Lehrbuch des Oshikuanjama (Bantu-Sprache in deutsch-südwest Afrika); Stuttgart \& Berlin, W. Spermann.

BUTAYE, R. - 1909 - Dictionnaire Kikongo-Français, Français-Kikongo, Roulers, Impr. Ed., Jules de Meester.

CAHEN, L. \& LEPERSONNE, J. - 1948 - Notes sur la Géomorphologie du Congo Occidental, Tervuren, A.M.C.B., Sciences Géologiques, Vol. 1.

CLERCQ, Aug. DE - 1960 - Dictionnaire Tshiluba-Français, Léopoldville, Impr. de la Société Missionnaire de St-Paul.

CORNET, J. - 1978 - Pierres sculptées du Bas-Zaïre, Catalogue d'exposition au Centre de Commerce International du Zaïre, Kinshasa, I.M.N.Z.

1981 - In the four moments of the sun, Catalogue, Washington, Board of Trustees, National Gallery of Art, pp. 221-224.

COUPEZ, A. - 1955 - Esquisse de la langue holoholo, Tervuren, A.M.R.C.B., Série in 8, Sciences de l'Homme, Linguistique, Vol. 12.

CURTIN, Ph. D. - 1969 - The Atlantic slave trade. A census, Madison, Milwaukee \& London, The University of Wisconsin Press.

CURTIN, Ph. D. et VANSINA, J. - 1964 - Sources of the nineteenth Atlantic slave trade, Journal of African History, Londres, T.V., Col. 2, pp. 185-208.

CUVELIER, J. - 1946 - L'ancien Royaume de Congo, Bruxelles, Desclée de Brouwer.

1953a - Documents sur une mission française au Kakongo 1766-1776, Bruxelles ; I.R.C.B., Mémoire.

1953b - Relations sur le Congo du Pere Laurent de Lucques (1700-1717), Bruxelles ; I.R.C.B., Section des Sciences morales et politiques, Mémoire, Coll. in $8^{\circ}$, t. XXXII, Fasc. 2, Série historique.

DELCOMMUNE, A. - 1922 - Vingt années de vie africaine, Récits de voyages, d'aventures et d'exploration au Congo Belge, Bruxelles, Veuve Ferdinand Larcier.

EKHOLM, K. - 1972 - Power and prestige. The riser and fall of the Kongo kingdom, Uppsala, Shriv Service AB.

FORTEMS, G. - 1960 - La densité de la population dans le Bas-Fleuve et le Mayumbe, Bruxelles, A.R.S.O.M., Sciences naturelles et médicales, Mémoire in $8^{\circ}$, t. XI, Fasc. 4.

GUSIMANA, B. - 1972 - Dictionnaire Pende-Français, Bandundu, Ceeba Série III, Vol. 1.

GUTHRIE, M. - 1948 - The classification of the Bantu languages, Londres, Gregg International publishers, Vol. 1-4.

HANNAN, M. - 1974 - Standard Shona dictionary, Salisbury, Mardon Printers.

HEUSCH, L. de - 1954 - Autorité et Prestige dans la société Tetela, Zaïre, 10, pp. 1011-1027.

1963 - Afrique Noire, L'Art et les Sociétés primitives a travers le monde, Paris, Hachette, pp. 13-115. HORTON, A.E. - 1953 - A dictionary of Luvale, El Monte, California, Rahn Brothers printing \& lithography Co. 
IRLE, J. - 1917 - Deutsch-Herero Wörterbuch, Hamburg, L. Friederichsen \& Co.

JADIN, L. - 1961 - Le Congo et la Secte des Antoniens. Restauration du Royaume sous Pedro IV et la "Saint Antoine" congolais (1694-1718), Bruxelles, Rome, extrait du B.I.H.B.R., Fasc. XXXIII.

1975 - L'ancien Congo et l'Angola. 1639-1655. D'après les archives romaines, portugaises, néerlandaises et espagnoles, Bruxelles-Rome, I.H.B.R., t. I, II, III.

KILTON, A. - 1985 - The Kingdom of Kongo, Oxford, Clarendon Press.

KITCHING, M.A. et BLACKLEDGE, G.R. - 1952 - A Luganda-English and English-Luganda dictionary, revised by Mulira E.M.K, et Ndawula, E.G.M., Kampala, Uganda, London, Great-Britain, Society for promoting Christian knowledge.

KJERSMEIER, C. - 1936 - Centre de style de la sculpture nègre africaine, Congo Belge, Paris, A. Morancé, Vol. I.

LAMAN, K.E. - 1936 - Dictionnaire Kikongo-Français avec une étude phonétique décrivant les dialectes les plus importants de la langue dite Kikongo, Bruxelles, mémoire de l'Institut Royal Belge.

MACGAFFEY, W. - 1970 - Custom and Government in the Lower Congo, Berkeley, Los Angeles, London, University of California Press.

MAES, J. - 1935 - Nouveaux dons au Musée du Congo Belge à Tervuren, Le Progrès, 20, pp. 133-135.

1938 - Kabila en grafbeelden uit Kongo, A.M.C.B., Ethnographie, Série VI, Tome II, fasc. 2.

MEULENBERG, J. - 1949 - Introduction à l'étude Pédologique des sols du territoire du Bas-Fleuve, Institut Royal Colonial, Section des Sciences naturelles \& médicales in $8^{\circ}$, t. XVIII, Fasc. 3.

MILLER, CM. - 1970 - Cokwe trade and conquest in the nineteenth century, Pre-colonial African trade, pp. 174-201.

MILLER, J.C. - 1975 - Legal Portuguese slaving from Angola. Some preliminary indications of volume and direction 1760-1830, Revue Française d'Histoire d'Outre-Mer, LXII, 226-227, pp. 135-176.

MISSIONS BENEDICTINES - 1950 - Vocabulaire Kisanga-Français, Abbaye de Saint-André-lez-Bruges.

MONTEIRO, J.J. - 1968 - Angola and the river Congo, London, Frank Cass and Co Ltd.

RODEGEM, F.M. - 1970 - Dictionnaire Rundi-Français, Tervuren, A.M.R.A.C., Série in 8º Sciences humaines, 69, XXVI.

SMITH, E.W. - 1907 - A handbook of the Ila language (commonly called the Seshukulumbwe), London, New York, Toronto, Oxford University Press.

SWARTENBROECKX, p. - 1973 - Dictionnaire Kikongo et Kituba-Français. Vocabulaire comparé des langages Kongo traditionnels et véhiculaires, Bandundu, Ceeba, Série III, Vol. 2.

TURNER, W.Y. - 1952 - Tumbuka-Tonga English dictionary, Blantyre, Nyasaland, The Hetherwick press, Church of Scotland mission.

VAN DER SLEEN, W.G.N. - 1958 - Ancient glass beads with special reference to the beads of East and Central Africa and the Indian Ocean, The journal of the Royal Anthropological Institute of Great Britain and Ireland, vol. 88, part II, July-December, pp. 203-216.

VANSINA, J. - 1959 - Esquisse de grammaire bushong, Tervuren, A.M. du C.B., Série in 8, Sciences de l'Homme, Linguistique, Vol. 23.

1965 - Les anciens royaumes de la savane, Léopoldville, Université Lovanium, I.R.E.S., Coll. Etudes Sociologiques, 1. 
1976 - Les anciens royaumes de la savane. Les Etats des savanes méridionales de l'Afrique Centrale, des origines à l'occupation coloniale, Kinshasa, PUZ ( $2^{\mathrm{e}}$ éd.).

VERLY, R. - 1955 - La statuaire de pierre au Bas-Congo (Bamboma-Mussurongo), Zaïre, IX, 5, pp. 451-528, pl.

VIANA, M.J. - 1961 - Dicionario de português-chi-yao e chi-yao-português, Laurenço Marques, Moçambique, Instituto de investigacâo cientifica.

\section{RÉSUMÉS}

The author has taken interest in the funerary monuments erected by the Bakongo ba Boma group which lives not very far from the town of Boma. Signs of contacts with Europeans appear on these monuments among which two different types can be distinguished: stone sculptures and ceramics. These richly decorated sculptures were used as commemorative monuments erected in honour of individuals entrusted with power or in memory of rich people. The use of stone or of ceramics is primarily related to the type of ground.

To glorify a recognized chief, it is determining that he is represented looking his best, surrounded by his court. This out-looking court is intended for the world of the living, it has its counterpart in another court inside the tomb which is entrusted with accompanying and serving the chief in the ancestors' village. There, we can find the chief's spouses and slaves, as well as his everyday and prestigious objects.

Tradition explains the function of these statues. They aim to embellish and identify certain tombs as long as possible; such is the role of elephant tusks, natural elements considered imposing since they come from an animal renouned for its tenacity and strength. Therefore, in order for a prestigious tomb to be identified, the synthesis of nature and culture expressed by the stone statue is of a great importance.

\section{AUTEUR}

\section{SHAJE TSHILUILA}

Institut des Musées Nationaux du Zaïre - Kinshasa - Zaïre 